\title{
Comparison of Geant 4, MCNP Simulation Codes of Studying Attenuation of Gamma rays through Biological Materials with XCOM and Experimental Data
}

\section{Medhat $\mathrm{ME}^{1,2 *}$, Shirmardi $\mathrm{SP}^{3}$ and Singh VP4}

${ }^{1}$ Experimental Nuclear Physics Department, Nuclear Research Centre, P.0.13759, Cairo, Egypt

${ }^{2}$ Institute of High Energy Physics, CAS, Beijing 100049, China

${ }^{3}$ Nuclear Technology and Science Research Institute (NSTRI), Tehran, Iran

${ }^{4}$ Department of Physics, Karnatak University, Dharwad, 580003, India

\begin{abstract}
Monte Carlo simulations have been carried out for studying mass attenuation coefficients for some vital biological materials (Blood, Bones, and Muscle) at different photon energies (59.5, 81.0, 356.5, 661.6, 1173.2 and $1332.5 \mathrm{keV}$ ). Appreciable variations are noted for attenuation coefficients by changing the photon energy and the chemical composition of the samples. The numerical simulations attenuation coefficients were compared with experimental data wherever possible and the XCOM theoretical data. The simulations show that the simulated mass attenuation coefficient values are very close to experimental values better than the other obtained theoretical data base for the same samples. The results indicate that MCNP and Geant 4 simulation codes can be applied to estimate mass attenuation for various biological materials at different energies. Monte Carlo method may be employed to make additional calculations on the photon attenuation characteristics of different biological sample for unknown experimental data.
\end{abstract}

Keywords: Attenuation coefficient; Monte Carlo; Biological materials

\section{Introduction}

The use of $\mathrm{X}$ - and gamma-rays in various fields such as in medicine, industry and agriculture is increasing; however the leakage and scattering of these rays can be harmful for human-beings. The leaked radiation may possibly interact with human being involve directly handling the nuclear facilities or public living nearby it. An understanding of how vital human organs such as blood (whole), bones and soft tissues interact with radiation is essential in the development of radiation protection and dosimetry. As such, a better understanding of X-ray interaction with human organs is necessary [1-4].

The mass attenuation coefficient $(\mu / \rho)$ is a measure of gamma ray absorption or scattering capability of element, compound or per unit mass. It is the fundamental parameter to derive many other parameters of radiation interaction, shielding and dosimetric interests. An extensive data is available in literatures relevant to mass attenuation coefficient and scattering cross-section for almost all elements, compounds as well as mixtures. Most of the obtained experimental data are compared with the theoretical tabulations used by XCOM program [5].

Geant 4 code is based on object-oriented programming and allows user to derive classes to describe the detector geometry, primary particle generator and physics processes models along electromagnetic, hadronic, and decay physics based on theory, materials and elements, experimental data or parameterizations. Most of physics processes models include multiple scattering, ionization, Bremsstrahlung, positron annihilation, photo-electric effect, Compton and Rayleigh scattering, pair production, synchrotron and transition radiation, Cherenkov effect, refraction, reflection, absorption, scintillation, fluorescence, and Auger electrons emission [6,7]. The Geant4 simulation toolkit covers a wide energy range starting from $250 \mathrm{eV}$ to the $\mathrm{TeV}$. The Geant 4 simulation is modeling of the photon attenuation through materials in computer environment gives flexibility and ease of use, instead of performing an experimental determination of mass attenuation coefficient of different composite materials. Recently one attempt has been used for simulation of attenuation coefficients [8].

Monte Carlo simulation is found an effective tool to calculate radiation interaction parameters in different types of compounds and mixtures for shielding and energy deposition in human organs and tissues. MCNP is a general purpose Monte Carlo code for transport of neutrons, photons and electrons. The user can apply up to second order surfaces (boxes, ellipsoids, cones, etc.) and fourth order torii to build a 3D geometry which can be filled with materials of arbitrary composition and density. Point, surface or volume sources of radiation can be defined, from which the mentioned particles are emitted with user specified probability distributions for energy and direction. The code then simulates the particle tracks and interactions with the materials, according to probability density distributions [9]. In the present study, the program used is based on Monte Carlo N Particle transport (MCNP-4C) code developed by the Los Alamos National Laboratory. The main objectives of the present study were to test the validity of MCNP and Geant4 based Monte Carlo simulations to demonstrate its success in studying radiation interactions through some vital biological materials (Blood, Bones, and Muscles). The two codes were applied in calculating mass attenuation and compared with their experimental measurements and the theoretical data obtained by XCOM program.

*Corresponding author: Medhat ME, Experimental Nuclear Physics Department Nuclear Research Centre, P.O.13759, Cairo, Egypt, Tel: 86 13693344534; E-mail: medhatme@ymail.com

Received June 04, 2014; Accepted August 07, 2014; Published August 13, 2014

Citation: Medhat ME, Shirmardi SP, Singh VP (2014) Comparison of Geant 4, MCNP Simulation Codes of Studying Attenuation of Gamma rays through Biological Materials with XCOM and Experimental Data. J Appl Computat Math 3: 179. doi: $10.4172 / 2168-9679.1000179$

Copyright: (c) 2014 Medhat ME, et al. This is an open-access article distributed under the terms of the Creative Commons Attribution License, which permits unrestricted use, distribution, and reproduction in any medium, provided the original author and source are credited. 
Citation: Medhat ME, Shirmardi SP, Singh VP (2014) Comparison of Geant 4, MCNP Simulation Codes of Studying Attenuation of Gamma rays through Biological Materials with XCOM and Experimental Data. J Appl Computat Math 3: 179. doi:10.4172/2168-9679.1000179

Page 2 of 5

\section{Monte Carlo Model and Simulations}

\section{MCNP simulation code}

Monte Carlo simulation is a general purpose tool for study of interaction of $\mathrm{X}-$, gamma, neutron and electrons with matter. It is based on the Monte Carlo method to solve the transport equation; furthermore, it can work on different modes of delivery that are capable to consider neutrons, electrons and photons, alone, or in pairs all three together. MCNP is a general-purpose, continuous-energy, generalized geometry, time-dependent, coupled neutron-photon-electron Monte Carlo transport code system. The MCNP-4C uses physics models for particle interactions and nuclear cross section libraries [10-12].

The simulated geometry consisted of a cubic model of $10 \times 10 \times 1$ $\mathrm{cm}^{3}$, the beam. The environment except the mentioned phantom was filled with the air. Finally, the cubic model was centered in an air sphere for variance reduction. Anything outside the air cube was considered void into which MCNP did not perform particle transport. Tally F2 was used to obtain MCNP-4C simulation data. This tally calculates flux in the cube sides for every source.

The MCNP source was modeled as a directional plane source in a vacuum. This source located at $10 \mathrm{~cm}$ away from the entry plane of the mentioned cube. The initial direction of gamma source was parallel to the beam axis. Simulations were performed with 100000 histories. All simulation data obtained by MCNP code were reported with less than $2 \%$ error.

\section{Geant4 simulation code}

The physics of Geant4 simulation depends on narrow beam geometry with the various photon energies. The experimental set-up of the simulation, consisting of a mono-energetic photon beam impinging on a sample is similar to earlier report for scintillation detectors [8]. The mass attenuation coefficients of investigated biological samples were determined by the transmission method according to Beer Lambert's law $\left(I=I_{o} e^{-\mu} m^{x}\right)$, where $\mathrm{I}_{0}$ and $\mathrm{I}$ are the incident and attenuated photon intensity, respectively, $\mu_{m}\left(\mathrm{~cm}^{2} \cdot \mathrm{g}^{-1}\right)$ is the mass attenuation coefficient and $x$ is the thickness of the slab. The thickness of the target is optimized according to the energy of the incident beam, to avoid that all the photons are absorbed in the target or traverse the slab without interacting. The primary photons emerging unperturbed from the slab are counted. The energy range of incident photons varied between 1 $\mathrm{keV}$ and $100 \mathrm{GeV}$. Attenuation of photons is calculated by simulating all relevant physical processes and interactions before and after inserting the samples under investigation. Photon interactions include photo-electric effect, Compton scattering, pair production, Rayleigh scattering, and electrons interactions include Bremsstrahlung, multiple scattering and ionization. Atomic effects after photo-electric effect, as $\mathrm{X}$-rays emission and Auger effect are included. So it is possible to have a vertex from photoelectric effect [13]. A good agreement between Geant 4 model for electromagnetic processes and National Institute of Standards and Technologies (NIST) reference data has been reported recently [13]. This statistical analysis estimated quantitatively the compatibility of Geant4 electromagnetic models with reference data and highlighted the respective strengths. The Geant 4 models are found in good agreement with the NIST reference data.

\section{XCOM Software}

The $\mu / \rho$ values of biological samples were calculated by mixture rule $\left((\mu / \rho)_{\text {composite }}=\sum^{n} w_{i}(\mu / \rho)_{i}\right)$ where $w_{i}$ is the proportion by weight and $(\mu / \rho)_{i}$ is mass attenuation coefficient of the $\mathrm{i}^{\text {th }}$ element by using XCOM (5). The uncertainties in $\mu / \rho$ values is about $1 \%$ for low- $Z$ $(1<\mathrm{Z}<8)$ in Compton region $(30 \mathrm{keV}$ to $100 \mathrm{MeV})$. It can generate cross-sections and attenuation coefficients for elements, compounds or mixtures in the energy range between $1 \mathrm{keV}$ and $100 \mathrm{GeV}$, in the form of total cross-sections and attenuation coefficients as well as partial cross-sections of the following processes: incoherent scattering, coherent scattering, photoelectric absorption and pair production in the field of the atomic nucleus and in the field of the atomic electrons. Below $30 \mathrm{keV}$ energy, the uncertainties are as much as $5-10 \%$ because of correction to experiments for high- $Z$ impurities and departure of Compton cross section from Klein-Nishina theory. Also above $100 \mathrm{MeV}$ photon energy, uncertainties in $\mu / \rho$ values may be $5-10 \%$. Uncertainties in photon energy absorption coefficient may be slightly greater values [14]. The gamma sources of photon energies above 5 $\mathrm{keV}$ are being used in medical, biological, industrial, radioactive source transportation and other shielding applications. Hence uncertainty in the result may not have any impact for practical applications.

\section{Materials and Method}

\section{Experimental setup}

The gamma ray spectrometry system consists of HPGe detector (Canberra model) coupled with analog digital converter (ADC), high voltage $5000 \mathrm{~V}$ with negative polarity and relative efficiency of $70 \%$. Genie 2000 software (Canberra Industries, Meriden, USA) with analyzer cart was used to record the intensity of the incident and the transmitted gamma rays. Energy calibration of the system was done initially with ${ }^{241} \mathrm{Am},{ }^{57} \mathrm{Co},{ }^{137} \mathrm{Cs}$, and ${ }^{60} \mathrm{Co}$ gamma ray point sources. Resolution (FWHM) of the system was $2.3 \mathrm{keV}$ at $1332.5 \mathrm{keV}$ gamma peaks of ${ }^{60} \mathrm{Co}$ point source kept at a distance of $10 \mathrm{~cm}$ in front of the detector face.

The detected spectra can be translated to ASCII and processed with custom-made programs based on ROOT. Automatic pulse shaping and pole-zero correction settings were used and the energy scale was calibrated using point radioactive sources. Incident and transmitted photons for each detector material were measured for sufficiently large fixed preset time to reduce statistical uncertainty. The measuring time is ranged from 5 to $10 \mathrm{~min}$ depending upon the photon energy and background noise. The background was counted in the same manner of measuring intensity of attenuated photons in the samples [8].

\section{Gamma source}

The sources were considered as planar ones with uniform distribution of radioactive material that emit gamma rays perpendicular to the front face of the shields. The emitted gamma rays were 59.5, 81.0, 356.5, 661.6, 1173.2 and $1332.5 \mathrm{keV}$ photons emitted by ${ }^{241} \mathrm{Am}$ $(2.78 \mathrm{GBq}),{ }^{133} \mathrm{Ba}(2.92 \mathrm{GBq}),{ }^{137} \mathrm{Cs}(3.14 \mathrm{GBq})$, and ${ }^{60} \mathrm{Co}(3.7 \mathrm{GBq})$ radioactive point source.

\section{Checking uniformity}

The uniformity of the measurements was checked by exposing different parts of samples to the incident gamma-ray beam. Stability and reproducibility of the procedure was tested before and after each run. The errors are attributed to the evaluation of the area under peaks, in the determination of the thickness of sample, the weighing of the sample, geometric factor, and intensity of the source, the systematic errors and the counting statistics.

\section{Specification of materials}

For specification of material in simulation, the user has to obtain 
Citation: Medhat ME, Shirmardi SP, Singh VP (2014) Comparison of Geant 4, MCNP Simulation Codes of Studying Attenuation of Gamma rays through Biological Materials with XCOM and Experimental Data. J Appl Computat Math 3: 179. doi:10.4172/2168-9679.1000179

Page 3 of 5

the elemental composition of blood, bones, and muscles on the base of their chemical composition and weight percent. The percentages by weight of the different elements for the investigated samples are presented in Table $1[15,16]$

\section{Gamma ray transmission factor}

The physics of simulation depends on narrow beam geometry with the various photon energies. The experimental set-up of the simulation, consisting of a mono-energetic photon beam impinging on a slab of one of the selected materials, the mass attenuation coefficients of investigated biological samples are determined by the transmission method according to Beer Lambert's law $\left(I=I_{o} e^{-\mu} m^{x}\right)$, where $\mathrm{I}_{0}$ and I are the incident and attenuated photon intensity, respectively, $\mu_{m}$ $\left(\mathrm{cm}^{2} \cdot \mathrm{g}^{-1}\right)$ is the mass attenuation coefficient and $x$ is the thickness of the slab. The thickness of the target is optimized according to the energy of the incident beam, to avoid that all the photons are absorbed in the target or traverse the slab without interacting. The primary photons emerging unperturbed from the slab are counted. The mass attenuation of photons for selected samples is calculated by simulating all relevant physical processes and interactions before and after inserting the investigated sample.

\section{Results and Discussion}

The mass attenuation coefficients of the biological samples were determined at 59.5, 81.0, 356.5, 661.6, 1173.2 and $1332.5 \mathrm{keV}$ photons by using gamma transmission method. The experimental error was estimated by combining errors for the product of linear attenuation coefficient and thickness, density and thickness measurements in

\begin{tabular}{|l|l|}
\hline Sample & Elemental Concentrations (\% weight) \\
\hline Blood & $\mathrm{H}(0.102), \mathrm{C}(0.11), \mathrm{N}(0.033), \mathrm{O}(0.745), \mathrm{Na}(0.001), \mathrm{P}(0.001), \mathrm{S}(0.002), \mathrm{Cl}(0.003), \mathrm{K}(0.002), \mathrm{Fe}(0.001)$ \\
\hline Bone & $\mathrm{H}(0.064), \mathrm{C}(0.278), \mathrm{N}(0.027), \mathrm{O}(0.41), \mathrm{Mg}(0.002), \mathrm{P}(0.07), \mathrm{S}(0.002), \mathrm{Ca}(0.147)$ \\
\hline Muscle & $\mathrm{H}(0.102), \mathrm{C}(0.123), \mathrm{N}(0.035), \mathrm{O}(0.729), \mathrm{Na}\left(8 \times 10^{-4}\right), \mathrm{Mg}\left(2 \times 10^{-4}\right)$ \\
\hline
\end{tabular}

Table 1: Chemical compositions of the investigated biological materials $[15,16]$.

\begin{tabular}{|c|c|c|c|c|c|c|c|c|c|c|c|c|}
\hline \multirow{4}{*}{ Energy (keV) } & \multicolumn{12}{|c|}{ Mass attenuation coefficients $\left(\mathrm{cm}^{2} \mathbf{g}^{-1}\right)$} \\
\hline & \multicolumn{4}{|c|}{ Blood } & \multicolumn{4}{|c|}{ Bone } & \multicolumn{4}{|c|}{ Muscle } \\
\hline & \multicolumn{2}{|c|}{ Simulation } & \multirow{2}{*}{ хсом } & \multirow{2}{*}{ Exp. } & \multicolumn{2}{|c|}{ Simulation } & \multirow{2}{*}{ XCOM } & \multirow{2}{*}{ Exp. } & \multicolumn{2}{|c|}{ Simulation } & \multirow{2}{*}{ XCOM } & \multirow{2}{*}{ Exp. } \\
\hline & Geant 4 & MCNP & & & Geant 4 & MCNP & & & Geant4 & MCNP & & \\
\hline 59.5 & 0.199 & 0.19 & 0.206 & 0.19 & 0.276 & 0.252 & 0.278 & 0.24 & 0.2 & 0.189 & 0.206 & 0.19 \\
\hline 81 & 0.191 & 0.172 & 0.182 & 0.16 & 0.197 & 0.192 & 0.207 & 0.19 & 0.187 & 0.171 & 0.187 & 0.17 \\
\hline 356.5 & 0.111 & 0.109 & 0.11 & 0.1 & 0.1 & 0.105 & 0.107 & 0.09 & 0.098 & 0.109 & 0.11 & 0.09 \\
\hline 661.6 & 0.088 & 0.084 & 0.085 & 0.08 & 0.081 & 0.081 & 0.082 & 0.07 & 0.081 & 0.084 & 0.085 & 0.08 \\
\hline 1173.3 & 0.061 & 0.064 & 0.065 & 0.06 & 0.057 & 0.061 & 0.062 & 0.04 & 0.075 & 0.064 & 0.065 & 0.05 \\
\hline 1332.5 & 0.057 & 0.06 & 0.066 & 0.06 & 0.061 & 0.057 & 0.058 & 0.05 & 0.055 & 0.06 & 0.061 & 0.05 \\
\hline
\end{tabular}

Table 2: Calculated and measured mass attenuation coefficients of the some biological materials.

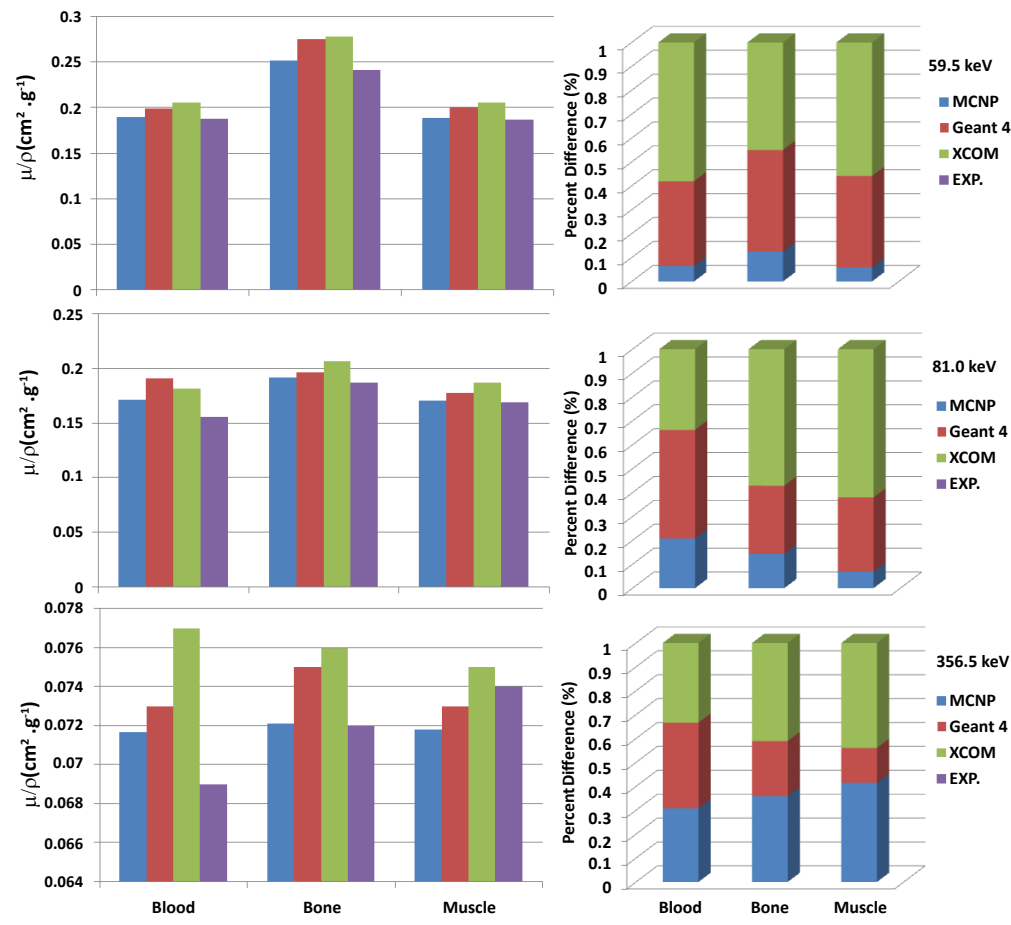

Figure 1: Mass attenuation calculated by MCNP, Geant4 and XCOM with respect to experiment according to relative deviation at 59.5, 81.0 and $356.5 \mathrm{keV}$ in biological samples. 
Citation: Medhat ME, Shirmardi SP, Singh VP (2014) Comparison of Geant 4, MCNP Simulation Codes of Studying Attenuation of Gamma rays through Biological Materials with XCOM and Experimental Data. J Appl Computat Math 3: 179. doi:10.4172/2168-9679.1000179

Page 4 of 5

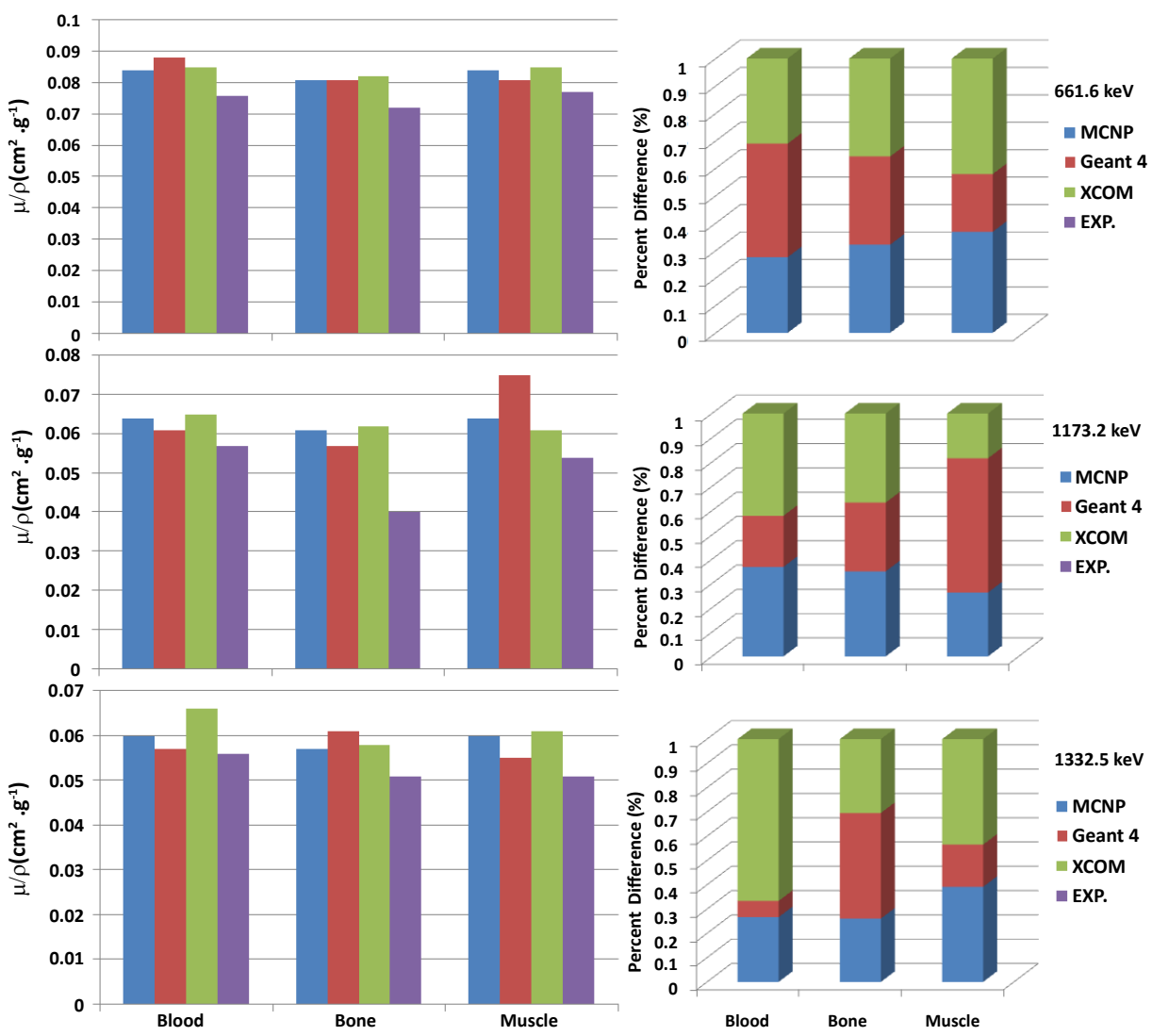

Figure 2: Mass attenuation calculated by MCNP, Geant4 and XCOM with respect to experiment according to relative deviation at $661.6,1173.2$ and $1332.5 \mathrm{keV}$ in biological samples.

quadrature. Calculations of the mass attenuation coefficients of all investigated biological samples were carried out by the XCOM program.

The results of our presented Geant 4 and MCNP simulation were compared with the theoretical values obtained using XCOM database and the obtained experimental results are shown in Table 2.

It is clear that there is satisfactory agreement between experiment and theory, although the experimental values tend to be lower than the theoretical estimation. Discrepancy in the values of the calculated and the experimental mass attenuation coefficients could be due to deviations from narrow beam geometry in the source-detector arrangements. The percent difference parameter between the experimental and theoretical values are lower than the corresponding values of XCOM program at different photon energies as shown in Figures 1 and 2. It is clear from this figures that percent difference values of MCNP and Geant 4 are lower than the corresponding values of XCOM for the different photon energies. The high values of XCOM return to the effect of chemical composition of biological samples and mixture rule. It is clear that the representative plot of the Monte Carlo generated data for mass attenuation coefficients as a function of incident photon energy and chemical composition. This trend was observed for each sample and this signifies that if we increase the energy of the incident photons we would obtain smaller attenuation, and therefore more penetration of the photons in the attenuator.

\section{Conclusion}

Monte Carlo simulation is a powerful tool in studying interaction of photons in materials. The applicability of this method is greatly dependent on the accuracy of geometry model, composition and density distribution of the sample matrix. The MCNP and Geant4 simulation were used to calculate mass attenuation coefficients of different wide types of materials in the $59.5-1332.5 \mathrm{keV}$ photon energy range. The simulations show that the calculated values of mass attenuation coefficients of biological samples are close to experimental values better than the values obtained by XCOM data base. These results conclude that MCNP and Geant4 Monte Carlo simulations can be applied to estimate mass attenuation coefficients for various attenuator and energies for which experimental results are not available.

\section{Acknowledgment}

The first author would like to thank all member of institute of high energy physics (IHEP) during hosting my postdoctoral fellowships presented by Chinese academy of science (CAS), China. This work was supported by the Chinese Academy of Sciences Fellowships for Young International Scientists.

\section{References}

1. Pravina P, Pawar PP, Bichile GK (2013) Studies on mass attenuation coefficient, effective atomic number and electron density of some amino acids in the energy range 0.122 to $1.330 \mathrm{MeV}$. Radi Phys and Chem.

2. Demir D, Turşucu A (2012) Studies on mass attenuation coefficient, mass energy absorption coefficient and kerma of some vitamins. Ann Nucl Energy 48: 17-20.

3. Antoniassi M, Conceicão ALC, Poletti ME (2011) Study of effective atomic 
Citation: Medhat ME, Shirmardi SP, Singh VP (2014) Comparison of Geant 4, MCNP Simulation Codes of Studying Attenuation of Gamma rays through Biological Materials with XCOM and Experimental Data. J Appl Computat Math 3: 179. doi:10.4172/2168-9679.1000179

Page 5 of 5

number of breast tissues determined using the elastic to inelastic scattering ratio. Instr Methods Phys Res A 652: 739-743.

4. Tomal A, Mazarro I, Kakuno EM, Poletti ME (2010) Experimental determination of linear attenuation coefficient of normal, benign and malignant breast tissues. Radiation Measurements 45: 1055-1059.

5. Berger MJ, Hubbell JH, Seltzer SM, Chang J, Coursey JS, et al. (2010) XCOM: photon cross sections database, NIST standard reference database (XGAM).

6. Agostinelli S, Allisonas J, Amakoe K, Apostolakisa J, Araujoaj H, et al. (2003) Geant4-a simulation toolkit. Nucl. Instrum Methods Phys Res A 506: 250-303.

7. CERN (2007) Geant4 collaboration physics reference manual.

8. Medhat ME, Wang Y (2013) Geant4 code for simulation attenuation of gamma rays through scintillation detectors. Ann Nucl Energy 62: 316-320.

9. Briesmeister JF (2000) MCNPTM-A General Monte Carlo N-Particle Transport Code Version 4C. RSICC COMPUTER CODE COLLECTION, ccc700 OAK RIDGE National Laboratory.

10. Shirmardi SP, Shamsaei M, Naserpour MM (2013) Comparison of photon attenuation coefficients of various barite concretes and lead by MCNP code, XCOM and experimental data. Ann. Nucl. Energy 55: 288-291.

11. Shultis JK, Faw RE (2010) An MCNP Primer. Kansas State University, Department of Mechanical and Nuclear Engineering.

12. Eakins J (2007) An MCNP-4C2 Determination of Gamma Source Shielding Health Protection Agency Center for Radiation, Chemical and Environmental Hazards Radiation Protection Division.

13. Amako K, Ibaraki KEK, Guatelli S, Ivanchenko VN, Maire M (2005) Comparison of Geant4 Electromagnetic Physics Models against the NIST Reference Data. IEEE transactions on Nuclear Science 52: 910-918.

14. Hubbell JH (1969) Photon cross section, attenuation coefficients, and energy absorption coefficients from $10 \mathrm{keV}$ to $100 \mathrm{GeV}$, NSRDS-NBS 29.

15. ICRU-44 (1989) Tissue Substitutes in Radiation Dosimetry and Measurement, Report 44 of the International Commission on Radiation Units and Measurements (Bethesda, MD).

16. Shivaramu (2002) Effective atomic numbers for photon energy absorption and photon attenuation of tissues from human organs. Medical Dosimetry 27: 1-9. 